\title{
Extracting Hadron Parameters from Dispersive Sum Rules
}

Wolfgang Lucha*

Institute for High Energy Physics, Austrian Academy of Sciences, Nikolsdorfergasse 18, A-1050

Vienna, Austria

E-mail: Wolfgang.Lucha@oeaw.ac.at

\section{Dmitri Melikhov}

Institute for High Energy Physics, Austrian Academy of Sciences, Nikolsdorfergasse 18, A-1050 Vienna, Austria, and

D. V. Skobeltsyn Institute of Nuclear Physics, Moscow State University, 119991, Moscow, Russia E-mail: dmitri_melikhov@gmx.de

\section{Silvano Simula}

INFN, Sezione di Roma III, Via della Vasca Navale 84, I-00146, Roma, Italy

E-mail: simula@roma3.infn.it

Puzzled or surprised by the almost incredible accuracy occasionally claimed in the literature to be achievable for numerical outcomes of QCD sum-rule analyses, we scrutinized the usual procedure employed for the extraction of the parameters of individual bound states from dispersive sum rules by taking advantage of the exact solvability of a quantum-mechanical harmonic-oscillator model: It turns out that the determination of the ground-state parameters (that is, decay constant and form factor) by requiring independence from the Borel mass in its stability window does not necessarily yield their exact numerical values [1 -6]. For instance, the comparison of the sum-rule predictions for bound-state parameters with their numerical values known precisely in our harmonic-oscillator model reveals that standard sum-rule procedures underestimate the ground-state decay constant by some $4 \%$ and its form factor by almost $15 \%$; such systematic uncertainties cannot be inferred from our correlators' accuracy better than $1 \%$ in the window of Borel stability: they are uncontrollable.

8th Conference Quark Confinement and the Hadron Spectrum

September 1-6, 2008

Mainz, Germany

\footnotetext{
${ }^{*}$ Speaker.
} 
The idea behind this sequence of studies at quantum-physics level [1-6] is simple and elegant: For a harmonic-oscillator model defined by the nonrelativistic Hamiltonian $H=\mathbf{p}^{2} / 2 m+m \omega^{2} \mathbf{x}^{2} / 2$ correlators, such as the polarization $\Pi(E) \equiv\left\langle\mathbf{x}_{\mathrm{f}}=\mathbf{0}\left|(H-E)^{-1}\right| \mathbf{x}_{\mathrm{i}}=\mathbf{0}\right\rangle$, are known at both "hadron" and "quark" levels, where the counterpart of the operator product expansion in QCD is easily found; at "hadron" level, the Borel transform of $\Pi(E)$, with Borel mass $\mu$, is $\Pi(\mu)=\sum_{n=0}^{\infty} R_{n} \exp \left(-E_{n} / \mu\right)$ with (exact) energies $E_{0}=3 \omega / 2, E_{1}=7 \omega / 2, \ldots$ and decay constants $R_{n} \equiv\left|\Psi_{n}(\mathbf{x}=\mathbf{0})\right|^{2}$. To extract from the quark level the ground-state parameters $E_{0}$ and $R_{0}$ numerically, duality, i.e., equality of the continuum contributions above a threshold energy is assumed. Equating the remainders of "hadron" and "quark" expressions yields a sum rule. This is best illustrated graphically (Fig. 1). However, the threshold's $\mu$-dependence induces uncontrolled uncertainties into this approach; without specifying this dependence sum-rule predictions are not reliable but plagued by considerable systematic errors.
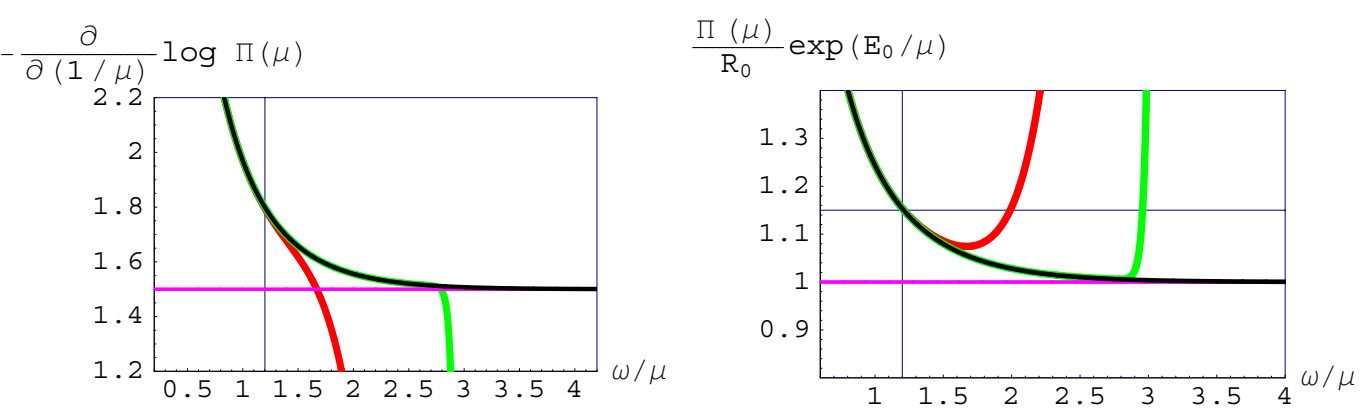

Figure 1: Extraction of the ground-state energy $E_{0}$ and decay constant $R_{0}$ of our model by either considering the exact expression (black) for $\Pi(\mu)$, or retaining 4 (red) and 100 (green) terms in its expansion for large $\mu$.

Acknowledgements. D. M. gratefully acknowledges financial support from the Austrian Science Fund (FWF) under projects P17692 and P20573, and from the RFBR under project 07-02-00551.

\section{References}

[1] W. Lucha, D. Melikhov, and S. Simula, Systematic uncertainties of hadron parameters obtained with QCD sum rules, Phys. Rev. D 76 (2007) 036002 [arXiv: 0705.0470 [hep-ph] ].

[2] W. Lucha, D. Melikhov, and S. Simula, Systematic errors of bound-state parameters obtained with SVZ sum rules, in QCD@ Work 2007 - International Workshop on Quantum Chromodynamics: Theory and Experiment, edited by P. Colangelo et al., AIP Conf. Proc. (AIP, Melville, New York, 2007), Vol. 964, p. 296 [arXiv: 0707.4123 [hep-ph] ].

[3] W. Lucha, D. Melikhov, and S. Simula, Study of systematic errors of bound-state parameters in SVZ sum rules, Phys. Atom. Nucl. 71 (2008) 1461.

[4] W. Lucha, D. Melikhov, and S. Simula, Can one control systematic errors of QCD sum-rule predictions for bound states?, Phys. Lett. B 657 (2007) 148 [arXiv : 0709.1584 [hep-ph] ].

[5] W. Lucha, D. Melikhov, and S. Simula, Systematic errors of bound-state parameters extracted by means of SVZ sum rules, in XII International Conference on Hadron Spectroscopy - Hadron 07, edited by L. Benussi et al., Frascati Phys. Ser. (INFN Laboratori Nazionali di Frascati, 2007), Vol. 46, p. 1109 [arXiv: 0712.0177 [hep-ph] ].

[6] W. Lucha, D. Melikhov, and S. Simula, Accuracy of bound-state form factors extracted from dispersive sum rules, HEPHY-PUB 865/08 (2008), arXiv: 0810.1920 [hep-ph] . 